\title{
Changes of inflammatory mediators and oxidative stress indicators in children with Henoch-Schönlein purpura and clinical effects of hemoperfusion in the treatment of severe Henoch-Schönlein purpura with gastrointestinal involvement in children
}

\author{
Ying Zhu ${ }^{1 *}\left(\mathbb{D}\right.$, Yang Dong ${ }^{1}$, Lin $\mathrm{Wu}^{1}$ and Fang Deng ${ }^{2}$
}

\begin{abstract}
Background: To explore the changes of inflammatory and oxidative stress responses in Henoch-Schönlein purpura (HSP) children, and further analyzed the therapeutic effects and mechanisms of hemoperfusion (HP) on HSP with severe gastrointestinal $(\mathrm{Gl})$ involvement.

Methods: There were 200 children with HSP were divided into three groups according to their clinical manifestations: 60 in HSP without Gl and renal involvement group, 60 in HSP with Gl involvement group, and 80 in HSPN group. The HSP with Gl involvement group was subdivided into conventional treatment $(n=30)$ and HP $(n=$ 30) groups. Thirty children who visited the department of children healthcare for healthy physical examinations from January to December 2017 were set as healthy control group. The IL-6 and TNF-a levels were detected by chemoluminescence method. The MDA, SOD and T-AOC levels were determined by thiobarbituric acid colorimetric method, hydroxylamine method and chemical colorimetry.

Results: Compared with healthy group, IL-6, TNF- $a$ and MDA levels in HSP were increased in each group, while SOD and T-AOC were decreased $(P=0.000)$. IL-6, TNF- $a$ and MDA levels in the HSPN group were the highest, SOD and T-AOC levels were the lowest $(P=0.000)$. Compared with those before treatment, IL-6, TNF-a and MDA levels in the conventional and HP groups were decreased and SOD and T-AOC levels were increased $(P=0.000)$. The changes in HP group were more significant than those in conventional group $(P<0.047)$. Compared with conventional group, glucocorticoid dosage and the occurrence rate of hematuria and/or proteinuria within 3 months were lower in HP group. $(P=0.000,0.004)$.

Conclusions: Inflammatory and oxidative stress may be involved in the acute phase of HSP children. The intensity of inflammatory and oxidative stress responses were related to the degree of renal involvement. HP can reduce glucocorticoid dosage and the rate of renal involvement in children with severe HSP with GI involvement. The mechanism may be related to the fact that HP can effectively remove IL-6, TNF-a, MDA in HSP children.
\end{abstract}

Keywords: Henoch-Schönlein purpura, Oxidative stress, Inflammatory mediators, Hemoperfusion, Children

\footnotetext{
*Correspondence: 114931468@qq.com

'Department of Nephrology, Anhui Provincial Children's Hospital, No. 39

Wangjiang East Road, Hefei 230051, China

Full list of author information is available at the end of the article
}

(c) The Author(s). 2019 Open Access This article is distributed under the terms of the Creative Commons Attribution 4.0 International License (http://creativecommons.org/licenses/by/4.0/), which permits unrestricted use, distribution, and reproduction in any medium, provided you give appropriate credit to the original author(s) and the source, provide a link to the Creative Commons license, and indicate if changes were made. The Creative Commons Public Domain Dedication waiver (http://creativecommons.org/publicdomain/zero/1.0/) applies to the data made available in this article, unless otherwise stated. 


\section{Background}

Henoch-Schönlein purpura (HSP) is one of the most common systemic vasculitis in childhood, which often invades the skin, gastrointestinal (GI) tract, joints, kidneys, a ccounting for an incidence of about 6-24/100, 000 per year [1-3]. HSP occurs to males more than to females, and the ratio between boys and girls is approximately 1.4:1 [1]. It is commonly observed in preschool and school-age children, and its peak incidence is observed between 2 and 6 years age-group, and $90 \%$ of the patients below10 years age [1-3]. Among them, about 50 to $75 \%$ of children had GI symptoms, and may also have refractory abdominal pain, GI bleeding, and may be accompanied by complications such as intestinal obstruction, intussusception and intestinal perforation and others in some severe cases [4-6], which in turn affected the early prognosis and remains difficult to treat. About 20 to $60 \%$ of the patients have renal involvement, which is known as Henoch-Schönlein purpura nephritis (HSPN) [7-12], and the severity of renal involvement affects the long-term prognosis of children with HSP [8-12]. The manifestations of HSPN vary from asymptomatic hematuria and/or proteinuria to nephritis syndrome, nephrotic syndrome, and rapidly progressive glomerulonephritis [8-12]. Renal biopsy is recommended if proteinuria occurs in children with HSPN [7].

The exact etiology and pathogenesis of HSP have not yet been fully elucidated. The pathogenesis of HSP is often associated with abnormal expression of inflammatory cytokines [13-15], oxidative stress [16], abnormal glycosylation of IgA1 [17], and genetic factors [13, 18]. Inflammatory mediators such as interleukin-6 (IL-6) [19], tumor necrosis factor- $\alpha$ (TNF- $\alpha)$ [15] and lipid peroxidation product malondialdehyde (MDA) [16, 20] played an important role in the initiation of inflammatory responses in HSP. The therapeutic effects of simple rash and articular purpura remained relatively good [21]. Currently, the treatment of severe HSP with GI and renal involvement depends on glucocorticoids, but it still remains difficult to be effective in some refractory cases. This may be due to that the inflammatory mediators and the oxidative stress products could not be eliminated timely in HSP.

AS a new technology invented in recent years, Hemoperfusion (HP) is applied to eliminated the inflammatory mediators and circulating immune complexes in the blood of HSP patients through extracorporeal circulation $[20,22-25]$. However, the mechanisms of HP in the treatment of severe HSP still remained unclear. Therefore this study has tried to explore the changes of inflammatory responses and oxidative stress in HSP children and its corelation to organ injury, and to further the study of therapeutic effects and mechanisms of HP on severe HSP with GI involvement. By comparing the levels of IL-6, TNF- $\alpha$, MDA, superoxide dismutase (SOD), and total anti-oxidant capability (T-AOC) in the acute phase of HSP children with different clinical manifestations, and the correlation between IL- 6 , TNF- $\alpha$, MDA and renal pathological grade, as well as comparison of the changes of IL- 6 , TNF- $\alpha$, MDA, SOD and TAOC levels before and after HP treatment in children with severe HSP with GI involvement and the clinical outcomes. And this study has summarized the effect of HP on glucocorticoid dosage and renal involvement to provide theoretical basis for clinical HP treatment of severe HSP with GI involvement.

\section{Methods}

\section{Study subjects and inclusion criteria}

A total of 200 HSP cases from the Department of Nephrology, Anhui Provincial Children's Hospital from January 2016 to June 2018 were selected as study subjects. There were 125 males and 75 females, aged 3-16 years old (7.73 \pm 2.77 years). Grouping: (1) These patients were divided into 3 groups according to the clinical manifestations: 60 patients in the HSP without GI and renal involvement group, 60 patients in HSP with GI involvement group, and 80 patients in the HSPN group. Thirty children who visited the department of children healthcare for healthy physical examinations from January to December 2017 were set as healthy control group. (2) The HSP with GI involvement group was divided into two subgroups according to whether HP treatment was performed: children in the conventional treatment group (30 cases) only received medical treatment and those in the HP group (30 cases) received HP treatment (once a day for 3 consecutive days) from day 2 after admission on the basis of conventional treatment. Inclusion criteria were as follows: (1) The diagnostic criteria of HSP patients are based on the EULAR/PRINTO/PRES in 2008 [26]. The diagnostic criteria of HSPN are based on ISKDC in 1974 and Nephrology group, Chinese branch of pediatrics, Chinese medical association in 2016 [7, 27]. (2) All children were first-onset cases without history of HSP and other chronic diseases. No children used glucocorticoids and antioxidants before undergoing examinations. The HSP without GI and renal involvement was manifested as simple rash and/or mild arthralgia. The HSP with GI involvement was manifested as active bleeding of digestive tract and/or persistent abdominal pain with or without intestinal complications (such as intussusception, intestinal obstruction, intestinal perforation, pancreatitis). There were moderate to high levels of proteinuria $(>25$ $\mathrm{mg} / \mathrm{kg} \cdot \mathrm{d}^{-1}$ ) with/without hematuria in the HSPN group. (3) There was no occurrence of hematuria and proteinuria in the HSP without GI and renal involvement group and HSP with GI involvement group. Renal biopsy was performed for all HSPN patients. The study was approved by 
the Medical Research Ethics Committee of Anhui Provincial Children's Hospital, informed consent was obtained from all guardians of individual participants included in the study.

\section{Therapeutic regimen}

Patients in the acute phase were treated according to the treatment guidelines [1, 28]: methylprednisolone was used to inhibit the immune responses for articular and HSP with GI involvement in the acute phase. Children in the HSPN group were treated with methylprednisolone and/or immunosuppressive agents according to the disease condition, children with proteinuria were administrated with angiotensin converting enzyme inhibitor (ACEI) and angiotensin receptor blocker (ARB) drugs. Seldinger technique was applied in the HP treatment, and a single-needle double-lumen catheter was inserted into the femoral vein to establish a temporary vascular access. The JA-800A hemoperfusion machine (Zhuhai Lizhu Biomaterial Co., Ltd.) was used and the HA280 resin hemoperfusion device was selected for HP treatment. The treatment duration was $2 \mathrm{~h} /$ time.

\section{Observational indicators}

Gender, age, clinical parameters were recorded for all patients. For the HSP with GI involvement, the remission of rash, abdominal pain and digestive tract bleeding were observed before treatment $(\mathrm{d} 0)$ and on day 4 after treatment (d4) in the conventional group of children, as well as before HP treatment (d0) and on day 4 after HP treatment for 3 times (d4) in the HP group of children. The dosage of methylprednisolone, length of hospital stay, hospital costs and complications of HP as well as high dose methylprednisolone were summarized, the relapse of rash and/or abdominal pain and the appearances of hematuria and/or proteinuria were statistically analyzed. All patients were followed up for at least 3 months (at 2 weeks, 1 month, 2 months and 3 months after discharge). The follow-up was performed by return visit or telephone interview, and the discussion included information regarding control or relapse of rash and abdominal pain, and results of urine routines.

\section{Detection of IL-6, TNF- $a$, MDA, SOD and T-AOC levels}

Venous blood of $3 \mathrm{~mL} \times 2$ samples were collected before treatment from all HSP children, on day 4 (d4) after treatment to detect HSP with GI involvement children of the conventional group and HP group, and after physical examinations for the children in the healthy control group. One blood sample was centrifuged at $4000 \mathrm{r} / \mathrm{min}$ for $5 \mathrm{~min}$ at $4{ }^{\circ} \mathrm{C}$. The supernatant was stored at $-80^{\circ} \mathrm{C}$. The levels of serum IL- 6 and TNF- $\alpha$ were detected by chemiluminescence method (the kit was purchased from Siemens). The other blood sample was centrifuged at
$2000 \mathrm{r} / \mathrm{min}$ for $15 \mathrm{~min}$ at $4{ }^{\circ} \mathrm{C}$. The plasma was then separated (stored at $-80^{\circ} \mathrm{C}$ ) to detect the MDA levels (thiobarbituric acid colorimetric method), SOD levels (hydroxylamine method) and T-AOC levels (chemical colorimetry) (the kits were purchased from Nanjing Jiancheng Institute of Bioengineering).

\section{Kidney biopsy}

Eighty children with HSPN underwent renal histopathological examinations taking the inferior pole of the right kidney as the puncture point under the guidance of ultrasound. The kidney tissues were taken out for light microscopy, immunofluorescence and electron microscopy.

\section{Statistical analysis}

Data was processed using SPSS 17.0 software. The measurement data was expressed as means \pm standard deviation $\left({ }^{-} x \pm s\right)$. One-way analysis of variance was used for comparisons between multiple groups. The t-test was used for pair-wise comparisons between groups. The enumeration data was expressed as percentages, and the comparisons between the groups were performed by row $\mathrm{x}$ column table $\chi^{2}$ tests. The correlation tests between the two variables were analyzed by Pearson linear correlation analysis. $P<0.05$ was considered to be statistically significant.

\section{Results}

Demographics, clinical parameters in each HSP group and healthy control group

There were no significant differences in gender, age, complement3 (C3) and fibrinogen (Fib) between the groups. Compared with the health group, the levels of C-reactive protein (CRP) and immunoglobulin A (IgA) in each HSP group were higher $(P<0.02, P=0.000)$. Compared with the HSP without GI and renal involvement group, the levels of white blood cell count (WBC) were higher, while albumin (ALB) were lower in the HSP with GI and HSPN groups $(P=0.000, P<0.001)$. Compared with the HSPN group, the levels of serum creatinine (Scr) were lower in HSP without GI and renal involvement and HSP with GI groups $(P=0.011,0.041)$ (Table 1$)$.

\section{Clinical parameters and biopsy results in HSPN patients}

No significant difference were found in age, gender, $24 \mathrm{~h}$ urine protein excretion (24hUPro) and urine red blood cell counts (URBC) in HSPN group with different pathological grades (Table 2).

\section{Changes in inflammatory indicators and oxidative stress indicators in healthy control group and each group with HSP}

The blood inflammatory mediators (IL-6 and TNF- $\alpha$ ), lipid peroxidation products (MDA), and antioxidant 
Table 1 Comparison of demographics, clinical parameters between each HSP group and healthy control group [cases(\%), $x \pm s$ ]

\begin{tabular}{llllll}
\hline & HSP-Gl and renal involvement $(n=60)$ & HSP $+\mathrm{Gl}$ involvement $(n=60)$ & HSPN $(n=80)$ & Healthy group $(n=30)$ & $P$ value \\
\hline Age (years) & $7.22 \pm 2.38$ & $7.58 \pm 3.17$ & $8.21 \pm 2.28$ & $8.31 \pm 2.05$ & 0.078 \\
Gender(M/F) & $35 / 25$ & $37 / 23$ & $53 / 27$ & $17 / 13$ & 0.727 \\
Abdominal pain & $0(0)$ & $60(100)$ & $48(60)$ & $0(0)$ & 0.000 \\
Arthralgia & $54(90)$ & $24(40)$ & $38(48)$ & $0(0)$ & 0.000 \\
WBC $\left(\times 10^{9} / \mathrm{L}\right)$ & $9.48 \pm 2.96$ & $14.71 \pm 6.02$ & $12.99 \pm 6.65$ & $8.31 \pm 2.05$ & 0.000 \\
CRP(mg/L) & $6.25 \pm 7.22$ & $11.05 \pm 13.70$ & $10.82 \pm 12.33$ & $3.13 \pm 2.41$ & 0.001 \\
ALB $(\mathrm{g} / \mathrm{L})$ & $43.46 \pm 3.74$ & $39.66 \pm 6.57$ & $37.68 \pm 6.83$ & $45.44 \pm 3.27$ & 0.000 \\
$\mathrm{SCr}(\mu \mathrm{mol} / \mathrm{L})$ & $29.2 \pm 6.89$ & $29.3 \pm 9.63$ & $34.13 \pm 11.27$ & $29.14 \pm 4.32$ & 0.002 \\
$\operatorname{lgA}(\mathrm{g} / \mathrm{L})$ & $2.08 \pm 1.00$ & $1.93 \pm 0.65$ & $2.21 \pm 0.82$ & $0.93 \pm 0.14$ & 0.000 \\
$\mathrm{C} 3(\mathrm{~g} / \mathrm{L})$ & $1.05 \pm 0.23$ & $1.07 \pm 0.26$ & $1.02 \pm 0.26$ & $1.08 \pm 0.18$ & 0.664 \\
Fib $(\mathrm{g} / \mathrm{L})$ & $2.73 \pm 0.60$ & $2.86 \pm 1.98$ & $2.43 \pm 0.76$ & $2.65 \pm 0.47$ & 0.179 \\
\hline
\end{tabular}

enzyme systems (SOD and T-AOC) showed statistically significant differences in each group $(P=0.000)$. The levels of IL- 6 , TNF- $\alpha$ and MDA in the acute phase of HSP children in each group were significantly higher than those in the healthy group, and the levels of SOD and $\mathrm{T}-\mathrm{AOC}$ were significantly lower than those in the healthy group $(P=0.000)$. Compared with the HSP without GI and renal involvement group, HSP with GI involvement group and healthy group, the levels of IL-6, TNF- $\alpha$ and MDA in the HSPN group were significantly higher $(P=0.000)$, while the levels of SOD and T-AOC were significantly lower $(P=0.000)$. Compared with the HSP without GI and renal involvement group and healthy group, the levels of IL-6, TNF- $\alpha$ and MDA in the HSP with GI involvement group were higher, while the levels of SOD and T-AOC were significantly lower $(P=0.000)$. Compared with the healthy group, the levels of IL-6, TNF- $\alpha$ and MDA in the HSP without GI and renal involvement group were higher, and the levels of SOD and T-AOC were significantly lower $(P=0.000)$, (Table 3$)$.
Correlation analysis of blood IL- 6 , TNF- $a$, MDA levels and renal pathological grade in HSPN group

The levels of IL-6, TNF- $\alpha$ and MDA in the HSPN group of patients were positively correlated with the degree of pathological grade $(r=0.843,0.875,0.070, P=0.000$, $0.000,0.003)$, see Figs. 1, 2, 3.

\section{Demographics, clinical parameters in the conventional group and HP group of HSP with GI involvement}

No significant difference in age, gender and the severity of disease was observed between the conventional treatment group and the HP group of the HSP with GI involvement (Table 4).

In the HSP with GI involvement, on day 4 after treatment, the proportion of patients with disappeared GI symptoms in the HP group was higher than that in the conventional group, and the difference was statistically significant $(P=$ 0.035). The time for disappearance of rash and GI symptoms in the HP group was less than that in the conventional group $(P=0.048,0.023)$. Compared with the conventional

Table 2 Clinical parameters and Histopathological examination in HSPN patients [cases(\%), ${ }^{-} x \pm s$ ]

\begin{tabular}{|c|c|c|c|c|c|c|}
\hline & 1 & Ila & $\mathrm{llb}$ & IIla & IIlb & $P$ value \\
\hline Cases(n) & $1(1)$ & $21(26)$ & $6(8)$ & $49(61)$ & $3(4)$ & \\
\hline Age(years) & 13 & $8.10 \pm 2.32$ & $7.67 \pm 2.73$ & $8.35 \pm 2.24$ & $7.67 \pm 3.06$ & 0.305 \\
\hline Gender(M/F) & $0 / 1$ & $11 / 10$ & $5 / 1$ & $35 / 14$ & $2 / 1$ & 0.269 \\
\hline Simplified proteinuria & $0(0)$ & $2(10)$ & $1(17)$ & $2(4)$ & $0(0)$ & \\
\hline Hematuria+proteinuria & $1(100)$ & $15(71)$ & $3(50)$ & $33(67)$ & $1(33)$ & \\
\hline Acute nephritis & $0(0)$ & $2(10)$ & $1(17)$ & $8(16)$ & $0(0)$ & \\
\hline Nephrotic syndrome & $0(0)$ & $2(10)$ & $1(17)$ & $6(12)$ & $2(67)$ & \\
\hline 24hUPro(mg/kg. $\left.\mathrm{d}^{-1}\right)$ & 25.68 & $43.17 \pm 35.98$ & $55.22 \pm 23.67$ & $52.01 \pm 43.80$ & $53.46 \pm 25.32$ & 0.876 \\
\hline URBC(/HP) & 12.00 & $77.35 \pm 140.05$ & $192.42 \pm 298.38$ & $130.77 \pm 304.42$ & $8.17 \pm 12.67$ & 0.783 \\
\hline
\end{tabular}


Table 3 Changes of blood inflammatory indicators and oxidative stress indicators in each HSP group and healthy control group $(-x \pm s)$

\begin{tabular}{|c|c|c|c|c|c|}
\hline & HSP-GI and renal involvement $(n=60)$ & $\mathrm{HSP}+\mathrm{Gl}$ involvement $(n=60)$ & $\operatorname{HSPN}(n=80)$ & Healthy group $(n=30)$ & $P$ value \\
\hline $\mathrm{IL}-6(\mathrm{pg} / \mathrm{mL})$ & $10.96 \pm 1.47^{a}$ & $18.37 \pm 1.84^{\mathrm{ab}}$ & $22.94 \pm 3.43^{\mathrm{ac}}$ & $2.31 \pm 0.56$ & 0.000 \\
\hline TNF-a(pg/mL) & $11.10 \pm 1.31^{\mathrm{a}}$ & $17.75 \pm 2.35^{\mathrm{ab}}$ & $22.26 \pm 3.35^{\mathrm{ac}}$ & $3.56 \pm 1.25$ & 0.000 \\
\hline $\mathrm{MDA}(\mathrm{nmol} / \mathrm{mL})$ & $2.04 \pm 0.12^{a}$ & $2.96 \pm 0.18 \mathrm{ab}$ & $3.33 \pm 0.42^{a c}$ & $1.39 \pm 0.24$ & 0.000 \\
\hline $\mathrm{SOD}(\mathrm{U} / \mathrm{mL})$ & $44.44 \pm 1.93^{\mathrm{a}}$ & $38.64 \pm 2.14 \mathrm{ab}$ & $34.12 \pm 2.73 a c$ & $56.84 \pm 4.62$ & 0.000 \\
\hline $\mathrm{T}-\mathrm{AOC}(\mathrm{U} / \mathrm{mL})$ & $8.62 \pm 0.73^{a}$ & $6.24 \pm 0.63^{a b}$ & $5.31 \pm 0.72^{a c}$ & $13.68 \pm 1.68$ & 0.000 \\
\hline
\end{tabular}

Note: a indicates comparison with the healthy control group, $P=0.00$

$\mathrm{b}$ indicates comparison with the HSP without $\mathrm{Gl}$ and renal involvement group, $P=0.000$

c indicates comparison with the HSP without GI and renal involvement group and HSP with Gl involvement group, $P=0.000$

group, the children in the HP group had lower glucocorticoid dosage and lower incidences of hematuria and/or proteinuria $(P=0.000,0.004)$, while hospital costs were significantly higher $(P=0.000)$. There were no significant differences in the length of hospital stay, recurrence rates of rash and abdominal pain between the two groups for three mounth $(P>0.05)$ (Table 4).

\section{Changes in inflammatory indicators and oxidative stress} indicators before and after treatment in the conventional group and HP group of HSP with GI involvement

In the HSP with GI involvement, the levels of IL-6, TNF- $\alpha$, MDA, SOD and T-AOC in the conventional group and the HP group showed no significant differences before treatment $(\mathrm{d} 0)(P>0.05)$. After treatment (d4), the levels of IL-6, TNF- $\alpha$ and MDA in the conventional group and the HP group of patients were lower than those before treatment. The SOD and T-AOC levels were recovered and increased compared with those before treatment, but the differences were still statistically significant compared with those in the healthy group $(P=0.000)$. After treatment $(\mathrm{d} 4)$, the levels of IL6 , TNF- $\alpha$ and MDA in the HP group were lower than those in the conventional group, while the levels of SOD and $\mathrm{T}-\mathrm{AOC}$ were higher than those in the conventional group $(P=0.000)$ (Table 5).

\section{Complications of HP and high dose methylprednisolone treatment}

There were 30 children treated with HP for a total of 90 times. During HP treatment, there were 3 cases of mild increase in blood pressure and 1 case of hypothermia. The patients with elevated blood pressure were selfrelief soon by dynamic monitoring, and the patient with

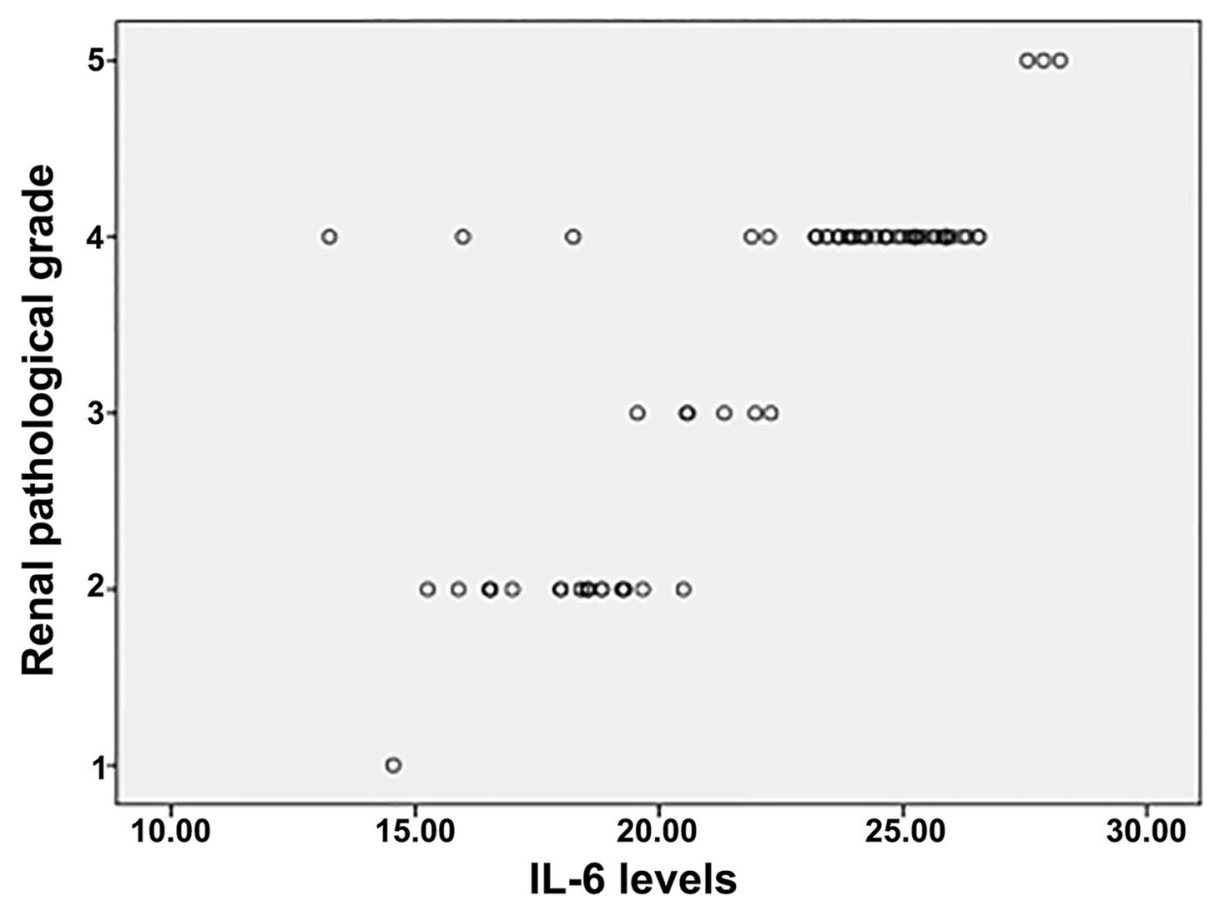

Fig. 1 Relationship between levels of serum IL-6 and renal pathological grade in children with HSPN 


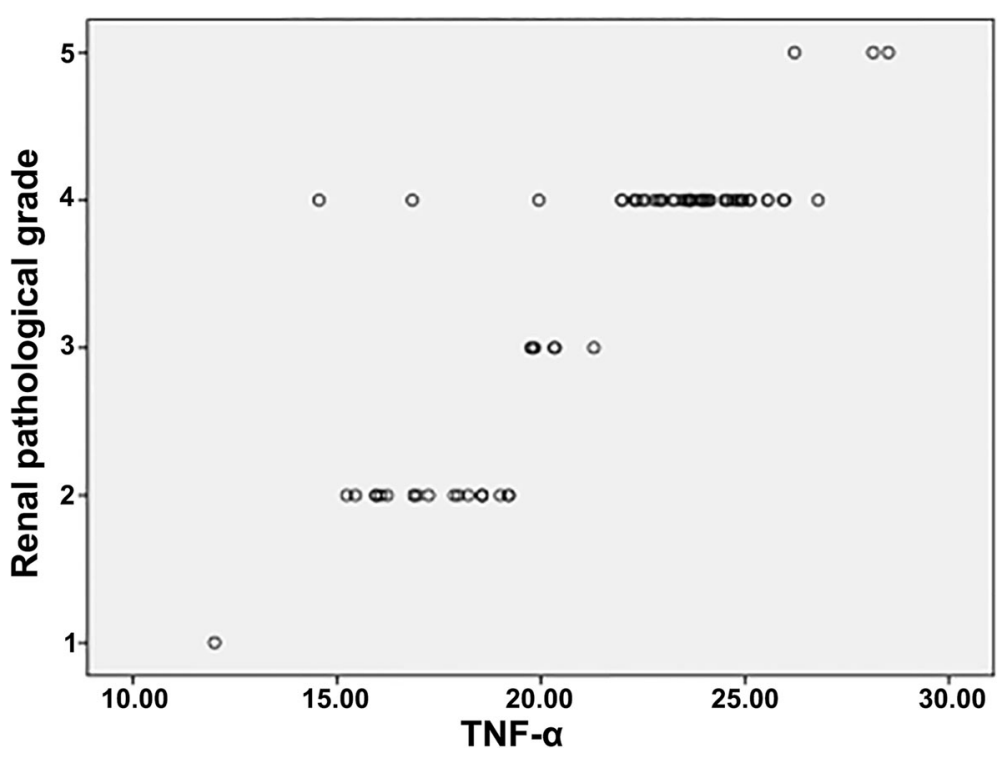

Fig. 2 Relationship between levels of serum TNF-a and renal pathological grade in children with HSPN

hypothermia got better after keeping warm. No complications such as hypotension, arrhythmia, bleeding, coagulation, thrombocytopenia, catheter related infection occurred. There were 30 children treated with high dose methylprednisolone for a total of 90 times. During the treatment, there were 12 cases of high intraocular pressure, 6 cases of hypertension, and 2 cases of tachycardia.

\section{Discussion}

This study was to investigate the changes of inflammatory and oxidative stress responses in the acute phase of
HSP children, the possible protection mechanism of HP for severe HSP with GI and renal involvement. The current research results show that IL-6, TNF- $\alpha$ and MDA might be involved in the acute phase of HSP. The intensity of inflammatory response and oxidative stress were closely related to the degree of illness in HSP. The curative effect of HP during the acute phase was better than that of medical treatment alone, and it can reduce glucocorticoid dosage and prevent renal involvement in severe HSP with GI involvement. The mechanism might be related to the fact that HP can quickly remove IL-6, TNF- $\alpha$, MDA in HSP children.

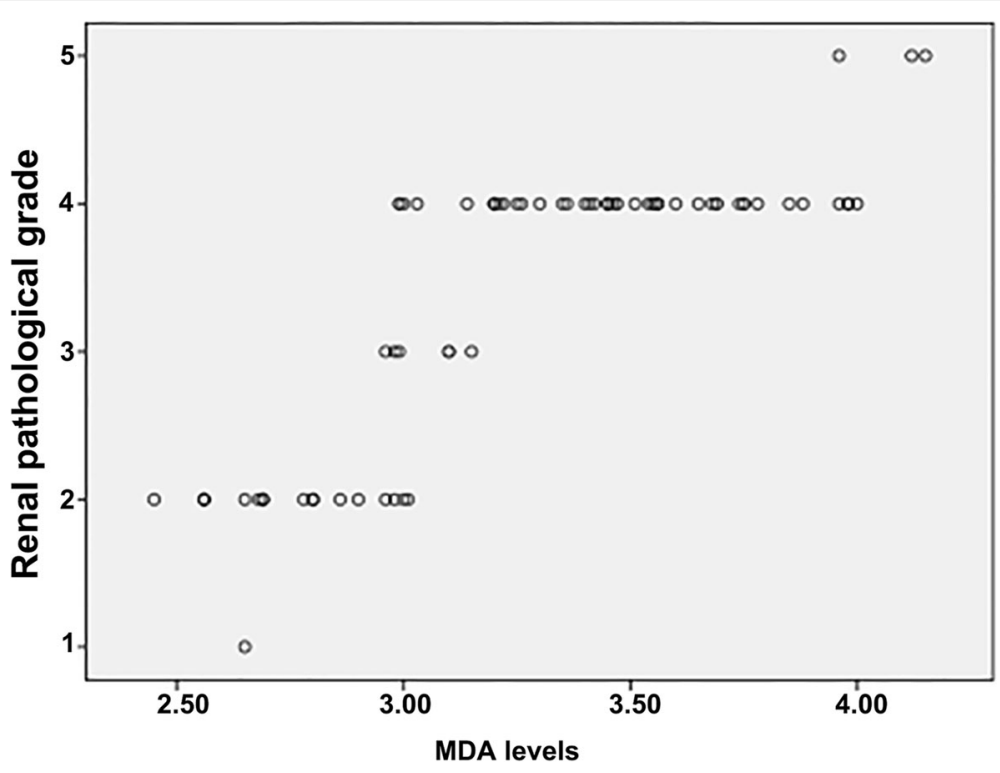

Fig. 3 Relationship between levels of plasma MDA and renal pathological grade in children with HSPN 
Table 4 Comparisons of clinical parameters, glucocorticoid dosage, costs, and renal damage and recurrence rate between the two groups $[\operatorname{cases}(\%),-x \pm s]$

\begin{tabular}{|c|c|c|c|}
\hline & Conventional group $(n=30)$ & HP group $(n=30)$ & $P$ value \\
\hline Age(years) & $7.40 \pm 2.97$ & $7.77 \pm 3.40$ & 0.658 \\
\hline Gender(M/F) & $17 / 13$ & $20 / 10$ & 0.596 \\
\hline Gl bleeding & 25(83) & 25(83) & 1.000 \\
\hline Persistent abdominal pain & $5(17)$ & $5(17)$ & 1.000 \\
\hline intussusception & 1(3) & $3(10)$ & 0.612 \\
\hline Intestinal obstruction & $0(0)$ & 1(3) & 1.000 \\
\hline Intestinal perforation & 1(3) & 1(3) & 1.000 \\
\hline Acute pancreatitis & 1(3) & 1(3) & 1.000 \\
\hline Arthralgia & $13(43)$ & $11(37)$ & 0.792 \\
\hline $\mathrm{WBC}\left(\times 10^{9} / \mathrm{L}\right)$ & $14.75 \pm 6.12$ & $14.66 \pm 6.03$ & 0.953 \\
\hline $\mathrm{CRP}(\mathrm{mg} / \mathrm{L})$ & $9.16 \pm 9.44$ & $12.93 \pm 16.90$ & 0.292 \\
\hline ALB $(g / L)$ & $40.46 \pm 6.40$ & $38.86 \pm 6.75$ & 0.351 \\
\hline $\operatorname{Scr}(\mu \mathrm{mol} / \mathrm{L})$ & $27.49 \pm 8.68$ & $31.02 \pm 10.35$ & 0.157 \\
\hline $\lg A(g / L)$ & $1.84 \pm 0.68$ & $2.02 \pm 0.62$ & 0.286 \\
\hline $\mathrm{C} 3(\mathrm{~g} / \mathrm{L})$ & $1.08 \pm 0.29$ & $1.04 \pm 0.23$ & 0.496 \\
\hline $\mathrm{Fib}(\mathrm{g} / \mathrm{L})$ & $3.25 \pm 2.69$ & $2.46 \pm 0.64$ & 0.125 \\
\hline Gl syndromes disappeared & $13(43)$ & $22(73)$ & 0.035 \\
\hline Time for rash disappearance(d) & $8.10 \pm 3.54$ & $6.57 \pm 2.18$ & 0.048 \\
\hline Time for digestive tract symptom disappearance(d) & $9.00 \pm 4.35$ & $6.33 \pm 4.51$ & 0.023 \\
\hline Daily glucocorticoid dosage (mg/kg) & $10.19 \pm 5.19$ & $4.74 \pm 1.44$ & 0.000 \\
\hline Hospital Costs(thousand yuan) & $17.23 \pm 8.60$ & $28.40 \pm 11.98$ & 0.000 \\
\hline Length of stay (d) & $16.5 \pm 6.5$ & $17.9 \pm 6.5$ & 0.435 \\
\hline Hematuria and/or proteinuria & 23(77) & $11(37)$ & 0.004 \\
\hline Recurrence of rah & $11(37)$ & $6(20)$ & 0.252 \\
\hline Recurrence of abdominal pain & $6(20)$ & $5(17)$ & 1.000 \\
\hline
\end{tabular}

The onset of HSP was associated with an imbalance of Th1/Th2 and Th2 preponderance activation, leading to increased secretion of Th2 cytokines. IL-6 is a core factor secreted by Th2 cells, and it can be used as an indicator of HSP activity. IL- 6 can stimulate the adhesion, proliferation and differentiation of glomerular mesangial cells of under synergistic action of other cytokines, which in turn lead to glomerular sclerosis [29, 30]. TNF$\alpha$ is a cytokine that is produced by mononuclear macrophages, and can stimulate immune-active cells to produce inflammatory factors such as interleukins. It can also cause structural changes in vascular endothelial cells, inhibit endothelial cell growth, and change the renal blood flow, leading to glomerular damage. Previous

Table 5 Changes of blood inflammatory indicators and oxidative stress indicators before and after treatment in the conventional group and HP group $\left({ }^{-} x \pm s\right)$

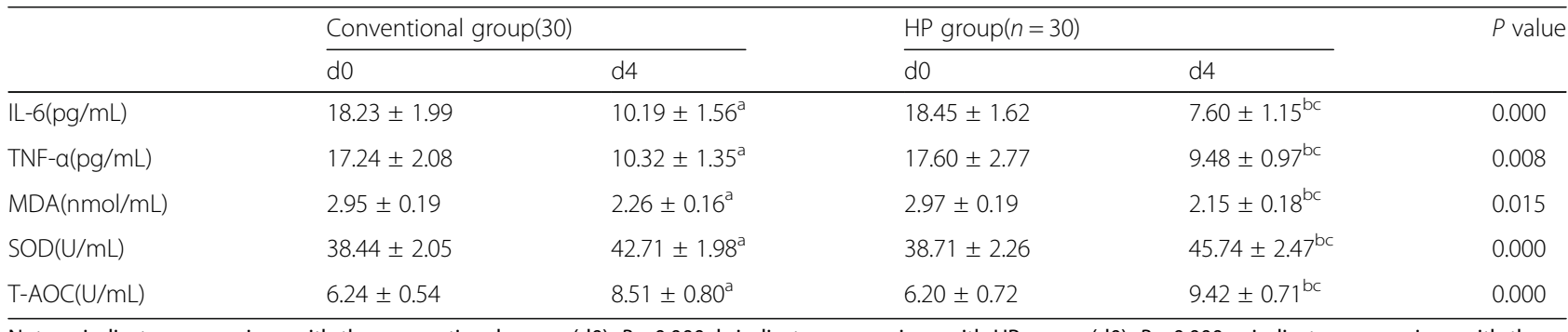

Note: a indicates comparison with the conventional group (d0), $P=0.000$. $\mathrm{b}$ indicates comparison with HP group (d0), $P=0.000$. $\mathrm{c}$ indicates comparison with the conventional group $(\mathrm{d} 4), P<0.047$ 
studies showed that the serum TNF- $\alpha$ levels were more significantly increased in children with HSPN, suggesting that TNF- $\alpha$ is closely related to renal injury in HSP [31, 32]. In this study, we found that children with HSP have excessive inflammatory response in vivo, and are closely related to the severity of the disease, the most obvious in HSPN, followed by severe HSP with GI involvement, the HSP without GI and renal involvement is the lightest.

After the damage of vascular endothelial cells, the neutrophils were activated, producing a large number of reactive oxygen species (ROS), further accelerating the progression of HSP [33]. The antioxidant enzyme systems such as T-AOC, SOD and others in the body maintain a dynamic balance of ROS production and clearance. When this balance was broken, lipid peroxidation occurs. MDA is a lipid peroxide that is produced by oxygen free radicals attacking the cell membranes. The levels of MDA were used as one of the indicators of lipid peroxidation degree, which indirectly reflects the degree of endothelial cell damage. Zhong Yanlan [20] reported that serum superoxide anion and MDA levels were significantly increased in children with severe HSP. Li Juan et al. [34] showed that MDA was correlated with the occurrence of HSPN. This study found that compared with the healthy group, the MDA in the acute phase of HSP children in each group were significantly increased, while the SOD and T-AOC were significantly decreased. In addition, the changes in the HSPN group were more obvious than those in the HSP with GI involvement group and the HSP without GI and renal involvement group, and the changes in the HSP with GI involvement group were more obvious than those in the HSP without GI and renal involvement group. This indicated that the intensity of lipid peroxidation in children with HSP was related to the clinical manifestations and severity of the illness.

Oxidative stress and inflammatory response play important roles in the mechanisms of renal damage and proteinuria $[35,36]$. Whether the kidney was affected or not and the extent of damage were considered as the most critical factors affecting the long-term prognosis of HSP. Further pathological biopsy of renal tissues helped to determine the degree of renal damage. HSPN was divided into VI according to the International Pediatric Kidney Disease Research Group [7, 27]. In the study, the indication of renal biopsy is more than moderate proteinuria with or without hematuria. Renal biopsies were performed in 80 patients, including 5 cases with simplified proteinuria, 53 cases with proteinuria and hematuria, 11 cases with acute nephritis and 11 cases with nephrotic syndrome. Renal pathology showed 1 case in grade I, 21 cases in grade IIa, 6 cases in grade IIb, 49 cases in grade IIIa, and 3 cases in grade IIIb. There were not statistically different in different pathological grades in $24 \mathrm{~h}$ urinary protein excretion and urinary red blood cell counts. This study found that the levels of IL-6, TNF- $\alpha$, and MDA in the HSPN group were positively correlated with the pathological grade. It was suggested that the inflammatory response and the oxidative stress might be involved in the pathological processes of renal damage during the acute phase of HSP. Meanwhile, the inflammatory response and the oxidative stress may aggravate the clinical progression of children. However, our study mainly focused on HSPN of class IIa and IIIa, and the highest class was IIIb. Changes in inflammatory response and the oxidative stress at higher pathological classes need to be further studied.

Glucocorticoids were mainly used for the treatment of HSP with GI involvement, but it was difficult to be cured in some cases as the inflammatory mediators and oxidative stress products cannot be eliminated in time [37]. Refractory or recurrent HSP has achieved a certain effect on the addition of immunosuppressive agents to glucocorticoid [38, 39]. However, these medicines have a slow onset and are limited in cases of severe acute attacks. HP is a new blood purification method developed in recent years. It used extracorporeal circulation to introduce the patient's blood into a perfusion device that contains a solid adsorbent to remove exogenous or endogenous toxic substances or morbid substance in the blood by adsorption. Then, the purified blood was returned to the body of the patient [40]. The HA-280 resin hemoperfusion device used a neutral macroporous adsorption resin as an adsorbent. It can effectively remove the inflammatory mediators, antibodies and circulating immune complexes, regulate and reconstruct the immune balance [37]. In this study, there were 50 cases of GI bleeding and 10 cases of persistent abdominal pain in HSP with GI involvement group, including 4 cases of intussusception, 2 cases of intestinal perforation, 2 cases of acute pancreatitis and 1 case of intestinal obstruction. The abdominal pain, digestive tract bleeding, rash and others were all relieved after treatment in both conventional group and HP group of the abdominal HSP, but the symptoms were relieved rapidly in the HP group. In children of the HP group, the glucocorticoid dosage was lower, and the IL- 6 and TNF- $\alpha$, and MDA were decreased more significantly, and the SOD and T-AOC was recovered and increased more significantly. The results suggested that the combination of medicines and HP in HSP with GI involvement improved the sensitivity of the body to medicines and reduced the dosage of glucocorticoids. The GI bleeding in children with HSP was one of the independent risk factors for HSPN [25]. Glucocorticoids can shorten the course of abdominal pain and GI bleeding in HSP, but cannot prevent the kidney involvement [28]. The results of this study suggested 
that the incidence of hematuria and/or proteinuria within 3 months of the disease was lower in the HP group, and the mechanisms might be related that by rapid elimination of IL- 6 , TNF- $\alpha$ and MDA by HP, preventing the damage of inflammatory mediators and lipid oxidative products on glomerular endothelial cells and improving the microcirculation of renal tissues to protect the renal function.

\section{Conclusions}

In conclusion, HP can reduce glucocorticoid dosage and the rate of renal involvement in children with severe HSP with GI involvement. The mechanism may be related to the fact that HP can effectively remove IL-6, TNF- $\alpha$, MDA in HSP children. But the treatment of HP is costly and invasive, limiting clinical use. However, HP treatment has no obvious complications, which is one of the safe and effective methods for the treatment of severe HSP with GI involvement, especially those with GI complications, high risk of kidney involvement and restriction of glucocorticoid usage during the acute phase. Nevertheless, its long-term efficacy should be further clarified in larger sample size by prospective randomized controlled clinical trials.

\section{Abbreviations \\ HP: Hemoperfusion; HSP: Henoch-Schönlein purpura; HSPN: Henoch- Schönlein purpura nephritis; MDA: Malondialdehyde; ROS: Reactive oxygen species; SOD: Superoxide dismutase; T-AOC: Total anti-oxidant capability}

\section{Acknowledgements}

Not applicable.

\section{Authors' contributions}

YZ carried out the studies, participated in collecting data, and drafted the manuscript. LW, FD and YZ performed the statistical analysis and participated in its design. YD, LW and FD helped to draft the manuscript. All authors read and approved the final manuscript.

\section{Funding}

This work was supported by the Anhui provincial department of health clinical medical technology application project (No.2008B07). The funders had no role in study design, data collection and analysis, decision to publish, or preparation of the manuscript.

\section{Availability of data and materials}

The datasets used and analysed during the current study are available from the corresponding author on reasonable request.

\section{Ethics approval and consent to participate}

All procedures performed in studies involving human participants were in accordance with the ethical standards of the institutional and/or national research committee and with the 1964 Helsinki declaration and its later amendments or comparable ethical standards. The study was approved by the Medical Research Ethics Committee of Anhui Provincial Children's Hospital, informed consent was obtained from all guardians of individual participants included in the study.

\section{Consent for publication}

Not applicable.

\section{Competing interests}

The authors declare that they have no competing interests.

\section{Author details}

'Department of Nephrology, Anhui Provincial Children's Hospital, No. 39 Wangjiang East Road, Hefei 230051, China. ${ }^{2}$ Department of Pediatrics, The First Affiliated Hospital of Anhui Medical University, Hefei 230022, China.

Received: 3 June 2019 Accepted: 24 October 2019

Published online: 04 November 2019

\section{References}

1. Li CF. Henoch-Schönlein purpura. Beijing: People's Medical Publishing House; 2015.

2. Chen AC, Lin CL, Shen TC, Li TC, Sung FC, Wei CC. Association between allergic diseases and risks of HSP and HSP nephritis: a population-based study. Pediatr Res. 2016;79:559-64.

3. Yang YH, Hung CF, Hsu CR, Wang LC, Chuang YH, Lin YT, et al. A nationwide survey on epidemiological characteristics of childhood HenochSchonlein purpura in Taiwan. Rheumatology (Oxford). 2005;44:618-22.

4. Basaran O, Cakar N, Uncu N, Celikel BA, Kara A, Cayci FS, et al. Plasma exchange therapy for severe gastrointestinal involvement of Henoch Schonlein purpura in children. Clin Exp Rheumatol. 2015;33:S-176-80.

5. Wang Y, Li S, Li JP. The experience of treatment and diagnosis for acute intussusceptions in children with Henoch Schönlein purpura. J Clin Pediatr Surg. 2015;5:422-4.

6. Li BD, Yan LB, Shen Y. Diagnosis and treatment of abdominal surgical complications of Henoch Schönlein purpura. Chin J Pediatr Surg. 2013;34: 310-1.

7. Nephrology group (2017) Chinese Medical Association. Evidence-based guidelines for the diagnosis and treatment of Henoch Schönlein purpura nephritis (2016). Zhonghua Er Ke Za Zhi. 2017;55:647-51.

8. Kaku Y, Nohara K, Honda S. Renal involvement in Henoch-Schonlein purpura: a multivariate analysis of prognostic factors. Kidney Int. 1998;53: 1755-9.

9. Sano H, lzumida M, Shimizu H, Ogawa Y. Risk factors of renal involvement and significant proteinuria in Henoch-Schonlein purpura. Eur J Pediatr. 2002; 161:196-201.

10. Rigante D, Candelli M, Federico G, Bartolozzi F, Porri MG, Stabile A. Predictive factors of renal involvement or relapsing disease in children with Henoch-Schonlein purpura. Rheumatol Int. 2005;25:45-8.

11. Chang WL, Yang YH, Wang LC, Lin YT, Chiang BL. Renal manifestations in Henoch-Schonlein purpura: a 10-year clinical study. Pediatr Nephrol. 2005; 20:1269-72.

12. Lee YH, Kim YB, Koo JW, Chung JY. Henoch-Schonlein Purpura in children hospitalized at a tertiary hospital during 2004-2015 in Korea: epidemiology and clinical management. Pediatr Gastroenterol Hepatol Nutr. 2016;19:175-85.

13. Yin W. Chen J. Immune pathogenesis of Henoch-Schönlein purpura in children. J Appl Clin Pediatr. 2017;32:1604-7.

14. Li B, Ren Q, Ling J, Tao Z, Yang X, Li Y. The change of Th17/Treg cells and IL-10/L-17 in Chinese children with Henoch-Schonlein purpura: A PRISMAcompliant meta-analysis. Medicine (Baltimore). 2019;98:e13991.

15. Yuan L, Wang Q, Zhang S, Zhang L. Correlation between serum inflammatory factors TNF-alpha, IL-8, IL-10 and Henoch-Schonlein purpura with renal function impairment. Exp Ther Med. 2018;15:3924-8.

16. Gurses D, Parlaz N, Bor-Kucukatay M, Kucukatay V, Erken G. Evaluation of oxidative stress and erythrocyte properties in children with henochshoenlein purpura. Iran J Pediatr. 2014;24:166-72.

17. Pillebout $E$, Jamin A, Ayari $H$, Housset P, Pierre M, Sauvaget V, et al. Biomarkers of IgA vasculitis nephritis in children. PLoS One. 2017;12: e0188718.

18. Xu H, Jiang G, Shen H, Pan Y, Zhang J, Li W, et al. The association between genetic variation in interleukin-10 gene and susceptibility to HenochSchonlein purpura in Chinese children. Clin Rheumatol. 2017;36:2761-7.

19. Kuret T, Lakota K, Zigon P, Ogric M, Sodin-Semrl S, Cucnik S, et al. Insight into inflammatory cell and cytokine profiles in adult IgA vasculitis. Clin Rheumatol. 2019;38:331-8.

20. Zhong YL, Dang XQ, He XJ. Effect and possible mechanism of hemoperfusion treatment for severe Henoch-Schönlein purpura. J Appl Clin Pediatr. 2013;28:1625-8.

21. Wu XC. Interpretation of evidence-based diagnosis and treatment recommendations for children with Henoch-Schönlein purpura. Chin J Pediatr. 2013;51:508-11. 
22. Shimizu T, Miyake T, Kitamura N, Tani M, Endo Y. Endotoxin adsorption: direct hemoperfusion with the polymyxin B-immobilized fiber column (PMX). Transfus Apher Sci. 2017;56:682-8.

23. Yamashita C, Moriyama K, Hasegawa D, Hara Y, Kuriyama N, Nakamura T, et al. Evidence and perspectives on the use of Polymyxin B-immobilized Fiber column Hemoperfusion among critically ill patients. Contrib Nephrol. 2018;196:215-22

24. Liu L, Huang YP, Fang XL, Zhang YY, Chen N, Hou HH. Effects of hemoperfusion treatment on serum IL-23 and IL-17 levels in children with Henoch-Schonlein purpure. Zhongguo Dang Dai Er Ke Za Zhi. 2015;17:796-9.

25. Ma DQ, Li Y, Han ZG, Zheng M, Xu N, Fan XJ. Analysis on kidney injuryrelated clinical risk factors and evaluation on the therapeutic effects of hemoperfusion in children with Henoch-Schonlein purpura. Eur Rev Med Pharmacol Sci. 2017;21:3894-9.

26. Ozen S, Pistorio A, lusan SM, Bakkaloglu A, Herlin T, Brik R, et al. EULAR/ PRINTO/PRES criteria for Henoch-Schonlein purpura, childhood polyarteritis nodosa, childhood Wegener granulomatosis and childhood Takayasu arteritis: Ankara 2008. Part II: final classification criteria. Ann Rheum Dis. 2010; 69:798-806.

27. Koskela M, Ylinen E, Ukonmaanaho EM, Autio-Harmainen H, Heikkila P, Lohi $J$, et al. The ISKDC classification and a new semiquantitative classification for predicting outcomes of Henoch-Schonlein purpura nephritis. Pediatr Nephrol. 2017;32:1201-9.

28. Chinese Medical Association, Editorial Board of Chinese. Evidence-based recommendations for the diagnosis and management in the children with Henoch-Schonlein purpura. Zhonghua Er Ke Za Zhi. 2013:51:502-7.

29. Kimura S, Takeuchi S, Soma Y, Kawakami T. Raised serum levels of interleukins 6 and 8 and antiphospholipid antibodies in an adult patient with Henoch-Schonlein purpura. Clin Exp Dermatol. 2013;38:730-6.

30. Sedaghat Z, Kadkhodaee M, Seifi B, Salehi E. Hind limb perconditioning renoprotection by modulation of inflammatory cytokines after renal ischemia/reperfusion. Ren Fail. 2016;38:655-62.

31. Sun AL. Significance of serum IL-6, IL-8 and TNF- $a$ expression in children with Henoch-Schönlein purpura. Chin J Pract Med. 2011;38:26-7.

32. Ha TS. The role of tumor necrosis factor-alpha in Henoch-Schonlein purpura. Pediatr Nephrol. 2005;20:149-53.

33. Chen T, Guo ZP, Zhang YH, Gao Y, Liu HJ, Li JY. Elevated serum heme oxygenase-1 and insulin-like growth factor-1 levels in patients with HenochSchonlein purpura. Rheumatol Int. 2011;31:321-6.

34. Li J, Fan CY, Wu K. Determination of urinary angiotensinogen and malondialdehyde and its clinical significance in children with HenochSchönlein purpuranephritis. J Clin Res. 2017:34:1836-8.

35. Ece A, Atamer Y, Gurkan F, Davutoglu M, Kocyigit Y, Tutanc M. Paraoxonase, total antioxidant response, and peroxide levels in children with steroidsensitive nephrotic syndrome. Pediatr Nephrol. 2005;20:1279-84.

36. El-Melegy NT, Mohamed NA, Sayed MM. Oxidative modification of lowdensity lipoprotein in relation to dyslipidemia and oxidant status in children with steroid sensitive nephrotic syndrome. Pediatr Res. 2008:63:404-9.

37. Dang $X Q$, Wu YB. Expert consensus interpretation of blood perfusion for children with severe Henoch-Schönlein purpura. Chin Pediatr Emerg Med. 2018;25:571-3.

38. Buscatti IM, Casella BB, Aikawa NE, Watanabe A, Farhat SCL, Campos LMA, et al. Henoch-Schonlein purpura nephritis: initial risk factors and outcomes in a Latin American tertiary center. Clin Rheumatol. 2018;37:1319-24.

39. Miray Kisla Ekinci R, Balci S, Serbes M, Duyuler Aycin G, Dogruel D, Ufuk Altintas D, et al. Recurrent Henoch Schonlein purpura without renal involvement successfully treated with methotrexate. Scott Med J. 2019; 64:74-7.

40. Gao Y, Deng HY. Blood perfusion in children (volume one). Beijing: Tsinghua University Publishing House; 2012.

\section{Publisher's Note}

Springer Nature remains neutral with regard to jurisdictional claims in published maps and institutional affiliations.

\section{Ready to submit your research? Choose BMC and benefit from:}

- fast, convenient online submission

- thorough peer review by experienced researchers in your field

- rapid publication on acceptance

- support for research data, including large and complex data types

- gold Open Access which fosters wider collaboration and increased citations

- maximum visibility for your research: over $100 \mathrm{M}$ website views per year

At BMC, research is always in progress.

Learn more biomedcentral.com/submissions 